\title{
Toward a physics design for NDCX-II, an ion accelerator for warm dense matter and HIF target physics studies
}

\author{
A. Friedman,${ }^{1, *}$ J. J. Barnard,${ }^{1}$ R. J. Briggs,${ }^{2}$ R. C. Davidson,${ }^{3}$ M. Dorf,${ }^{3}$ \\ D. P. Grote ${ }^{1}$ E. Henestroza, ${ }^{2}$ E. P. Lee, ${ }^{2}$ M. A. Leitner,${ }^{2}$ B. G. Logan ${ }^{2}$ \\ A. B. Sefkow ${ }^{3, \dagger}$ W. M. Sharp, ${ }^{1}$ W. L. Waldron, ${ }^{2}$ D. R. Welch, ${ }^{4}$ and S. S. $\mathrm{Yu}^{2}$ \\ ${ }^{1}$ Lawrence Livermore National Laboratory, Livermore CA USA \\ ${ }^{2}$ Lawrence Berkeley National Laboratory, Berkeley CA USA \\ ${ }^{3}$ Princeton Plasma Physics Laboratory, Princeton NJ USA \\ ${ }^{4}$ Voss Scientific, Albuquerque NM USA
}

(Dated: August 12, 2008)

The Heavy Ion Fusion Science Virtual National Laboratory (HIFS-VNL), a collaboration of LBNL, LLNL, and PPPL, has achieved 60-fold pulse compression of ion beams on the Neutralized Drift Compression eXperiment (NDCX) at LBNL. In NDCX, a ramped voltage pulse from an induction cell imparts a velocity "tilt" to the beam; the beam's tail then catches up with its head in a plasma environment that provides neutralization. The HIFS-VNL's mission is to carry out studies of Warm Dense Matter (WDM) physics using ion beams as the energy source; an emerging thrust is basic target physics for heavy ion-driven Inertial Fusion Energy (IFE). These goals require an improved platform, labeled NDCX-II. Development of NDCX-II at modest cost was recently enabled by the availability of induction cells and associated hardware from the decommissioned Advanced Test Accelerator (ATA) facility at LLNL. Our initial physics design concept accelerates a $\sim 30 \mathrm{nC}$ pulse of $\mathrm{Li}^{+}$ions to $\sim 3 \mathrm{MeV}$, then compresses it to $\sim 1$ ns while focusing it onto a mm-scale spot. It uses the ATA cells themselves (with waveforms shaped by passive circuits) to impart the final velocity tilt; smart pulsers provide small corrections. The ATA accelerated electrons; acceleration of non-relativistic ions involves more complex beam dynamics both transversely and longitudinally. We are using analysis, an interactive one-dimensional kinetic simulation model, and multidimensional Warp-code simulations to develop the NDCX-II accelerator section. Both LSP and Warp codes are being applied to the beam dynamics in the neutralized drift and final focus regions, and the plasma injection process. The status of this effort is described.

PACS numbers: 29.27.-a; 52.65.Cc; 52.65.Rr

Keywords: Accelerator, Fusion, Heavy-ion, Induction, Simulation, Particle-in-cell, Plasma, Beam

\footnotetext{
*Correspondence to: Dr. Alex Friedman, MS 47R0112, LBNL, One Cyclotron Road, Berkeley CA 94720; 510-486-5592; mailto:af@llnl.gov

${ }^{\dagger}$ Present affiliation: Sandia National Laboratories, Albuquerque, NM USA
} 
The Heavy Ion Fusion Science Virtual National Laboratory (HIFS-VNL), a collaboration of Lawrence Berkeley National Laboratory, Lawrence Livermore National Laboratory, and Princeton Plasma Physics Laboratory, is applying intense ion beams as drivers for basic studies of Warm Dense Matter (WDM) physics in uniformly heated foils driven by ions near the Bragg peak energy, and is studying their ultimate application for Inertial Fusion Energy (IFE). For an overview of this effort, see [1]; more information can be found in [2]. An emerging emphasis is on the novel properties of ion direct drive for inertial fusion energy [3]. On the Neutralized Drift Compression eXperiment (NDCX) at LBNL, a velocity "tilt" is imparted to the beam as it traverses an induction gap by a ramped voltage pulse; the beam's tail then catches up with its head in a plasma environment that provides needed neutralization. Using this technique, compression factors of 60 or greater have been achieved $[4,5]$. These studies are employing $\mathrm{K}^{+}$ions at $350 \mathrm{keV}$ and $\sim 25 \mathrm{~mA}$. Greater uniformity of energy deposition would be obtained by using ions that impinge on the target foil at an energy slightly above the Bragg peak, then slow in the target and exit at an energy slightly below that peak; this requires an improved facility, NDCX-II.

We have developed the basis for an attractive physics design for NDCX-II. Our goal is a machine concept capable of meeting the needs of the WDM research program described above and, after straightforward extension, of supporting a rich set of ionbeam-driven target experiments to explore fundamental aspects of ion direct drive. Here, we briefly outline the options considered, and then review the basic features of the concept selected as our baseline and our calculations in support of it. See also [6].

To generate an initial beam with a high line charge density, we plan to extract a long, high-current pulse from a diode at relatively high energy, and then pass the beam through a decelerating field to compress it. To understand this, consider a lossless steady injection: the current is constant along the axis, so the slower downstream beam has an increased line-charge density. The low-energy beam bunch is directed into a solenoid and matched into a Brillouin flow. The Brillouin equilibrium is independent of the energy if the relationship between the beam size $(a)$, solenoid magnetic field strength $(B)$ and line charge density $(\lambda)$ is such that $(B a)^{2}$ is proportional to $\lambda$. Such an "accel-decel" system serves as the front end of our physics design concept for NDCXII. To explore this type of injector, an experiment has been formulated [7] in which we would extract $\mathrm{a} \sim 1 \mu \mathrm{s}, 100 \mathrm{~mA} \mathrm{~K}{ }^{+}$beam at $160 \mathrm{keV}$, and decelerate it to $55 \mathrm{keV}$ $(\lambda \sim 0.2 \mu \mathrm{C} / \mathrm{m})$.

A study [8] of ion beam drivers for creating WDM conditions explored several approaches to accelerate and compress $30 \mathrm{nC}$ of $\mathrm{Li}^{+}$ions to $2.8 \mathrm{MeV}$ or more. Three options were considered, all beginning with an accel-decel injector producing a 100 $\mathrm{keV}$ beam: (a) a $3-\mathrm{m}$ electrostatic column (with $10 \mathrm{kV} / \mathrm{cm}$ gradient); (b) a sequence of pulse-line ion accelerator (PLIA) sections, running in the "snowplow" mode; and (c) an induction accelerator using cells from the decommissioned ATA accelerator at LLNL. All three concepts appeared feasible, but the maturity of the induction ap- 
proach and the existence of much of the hardware has led us to select that approach as our baseline. Research into the PLIA approach continues in the HIFS-VNL [9-11] and at CUHK [12]; a PLIA might serve as a front-end upgrade, or as an "afterburner" for higher energy.

The arrangement of induction cells and applied accelerating waveforms that we propose is novel, but the system is based on well-established technologies. It takes full advantage of the available ATA ferromagnetic cells and Blumleins. The system is compact, and relies heavily on passive circuit elements to provide the requisite waveform shaping. The adaptation from ATA, which accelerated electrons, has been nontrivial, because of the need to aggressively compress the ion pulse from its initial $\sim 500 \mathrm{~ns}$ duration to $\sim 1 \mathrm{~ns}$, as required for the WDM physics mission. The applied waveforms must simultaneously impose a head-to-tail velocity tilt, compensate for the beam space charge, and accelerate the beam.

Using a 1-D particle-in-cell simulation code designed for this purpose, we developed an acceleration schedule employing thirty ATA cells (twenty driven by the ATA Blumleins, plus ten with lower-voltage sources). To reduce the axial extent of the gap fringe fields, the $6.7-\mathrm{cm}$ radius of the ATA beam pipe is reduced to $4.0 \mathrm{~cm}$. This system accelerates a $\mathrm{Li}^{+}$beam (100 keV as injected, with a $67 \mathrm{~mA}$ flat-top) to $3.5 \mathrm{MeV}$ at $\sim 2 \mathrm{~A}$, and imparts an $8 \%$ tilt. After neutralized drift compression, about $75 \%$ of the $30 \mathrm{nC}$ beam charge crosses the focal plane in a 1-ns window, with a minimal pre-pulse. The current of the compressed beam (averaged over that window) is $\sim 23 \mathrm{~A}$, with a peak (averaged over a 0.1-ns window) of $\sim 32 \mathrm{~A}$ and an FWHM of $\sim 1$ ns.

A novel two-part strategy is employed to accelerate and compress the beam. In (roughly) the first half of the lattice, the pulse is rapidly compressed via "non-neutral drift compression." The beam transit time through an acceleration gap (including its axially extended fringe field) must be less than $70 \mathrm{~ns}$ for a high-voltage (up to 200 $\mathrm{kV}$ ) ATA Blumlein to be used as the pulser. Custom pulsers at lower voltage are required for longer pulses; to minimize the number of these, we use the volt-seconds of the first two cell blocks to impose a velocity tilt, with space between tilt cells for drift compression and longitudinal control. To avoid short-wavelength density irregularities, we choose the tilt-cell fields to maintain, insofar as possible, a linear velocity variation and a smooth density profile. We obtain the desired waveforms using a least-squares optimization that penalizes both nonlinearity and nonuniformity.

In the second half of the lattice, the beam is allowed to lengthen as it is accelerated, with only enough ramped pulses added to keep the duration under 70 ns. The initial compression is slowed by the increasing space-charge field; after the beam passes through a longitudinal waist, it begins to lengthen as a consequence of acceleration and space charge. Since the beam at the waist is shorter than the longitudinal extent of the gap fields, those fields cannot prevent this "bounce;" however, as the length increases, tilt cells can keep the beam duration from exceeding 70 ns. We find that two tilt cells in each block of five suffice. The modularity of these blocks is an attractive feature, 


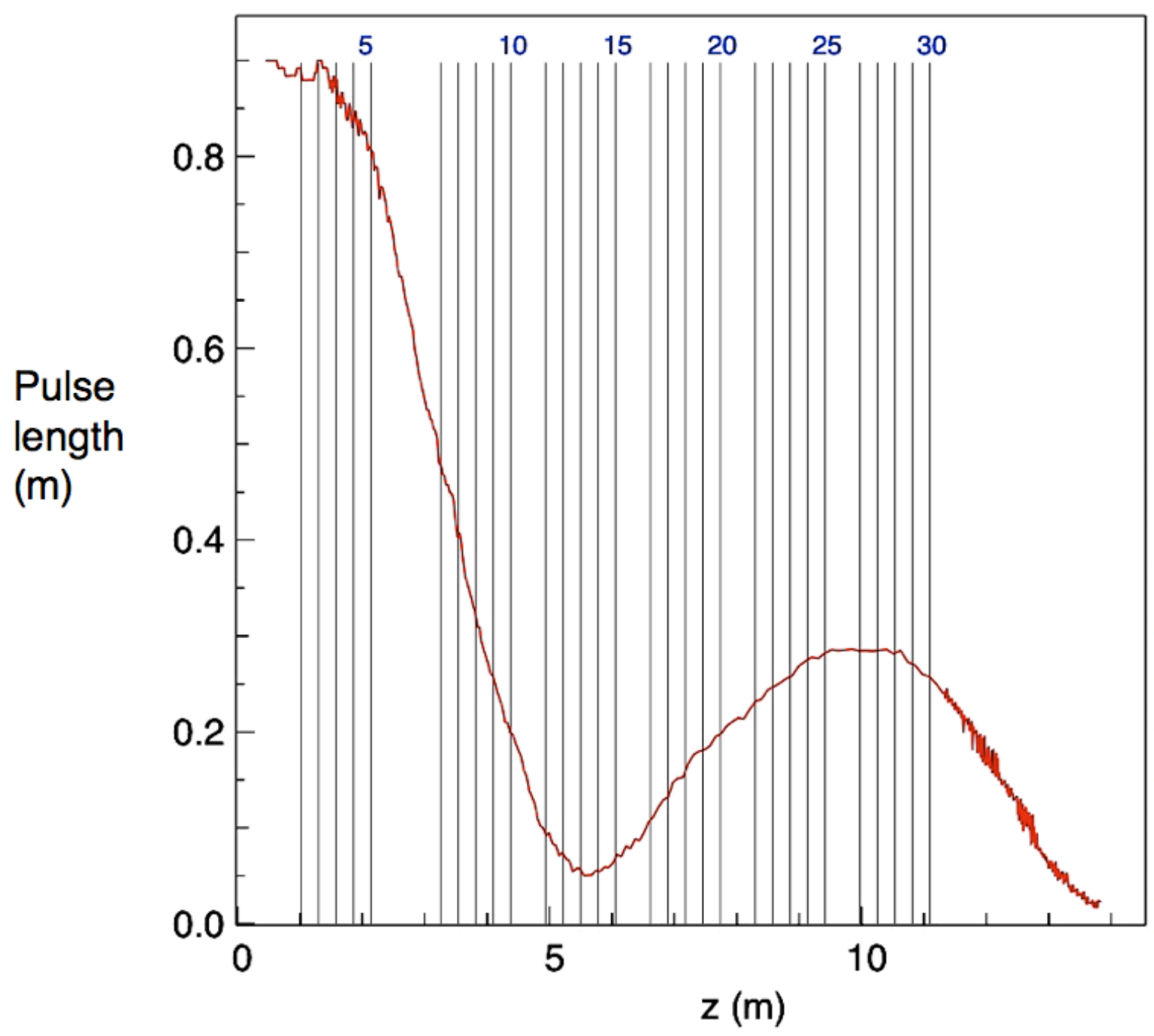

FIG. 1: Pulse length vs. axial coordinate $z$. Numbers label the accelerating gaps.

since more can be added if a higher final kinetic energy is desired. A final block with five ramped pulses applies the tilt for neutralized drift compression onto the target; this is another attractive feature, since no high-voltage "tilt core" is required. Figure 1 shows the evolution of the beam length, while Fig. 2 shows the evolution of the pulse duration.

All the waveforms in this acceleration schedule, except for the longitudinal-control fields or "ears" applied in the first two blocks, are simple enough to be formed with passive circuits in the "compensation boxes" that are attached to the ATA cells. For the case discussed here, all the tilt-cell waveforms in the final four cell blocks have been 


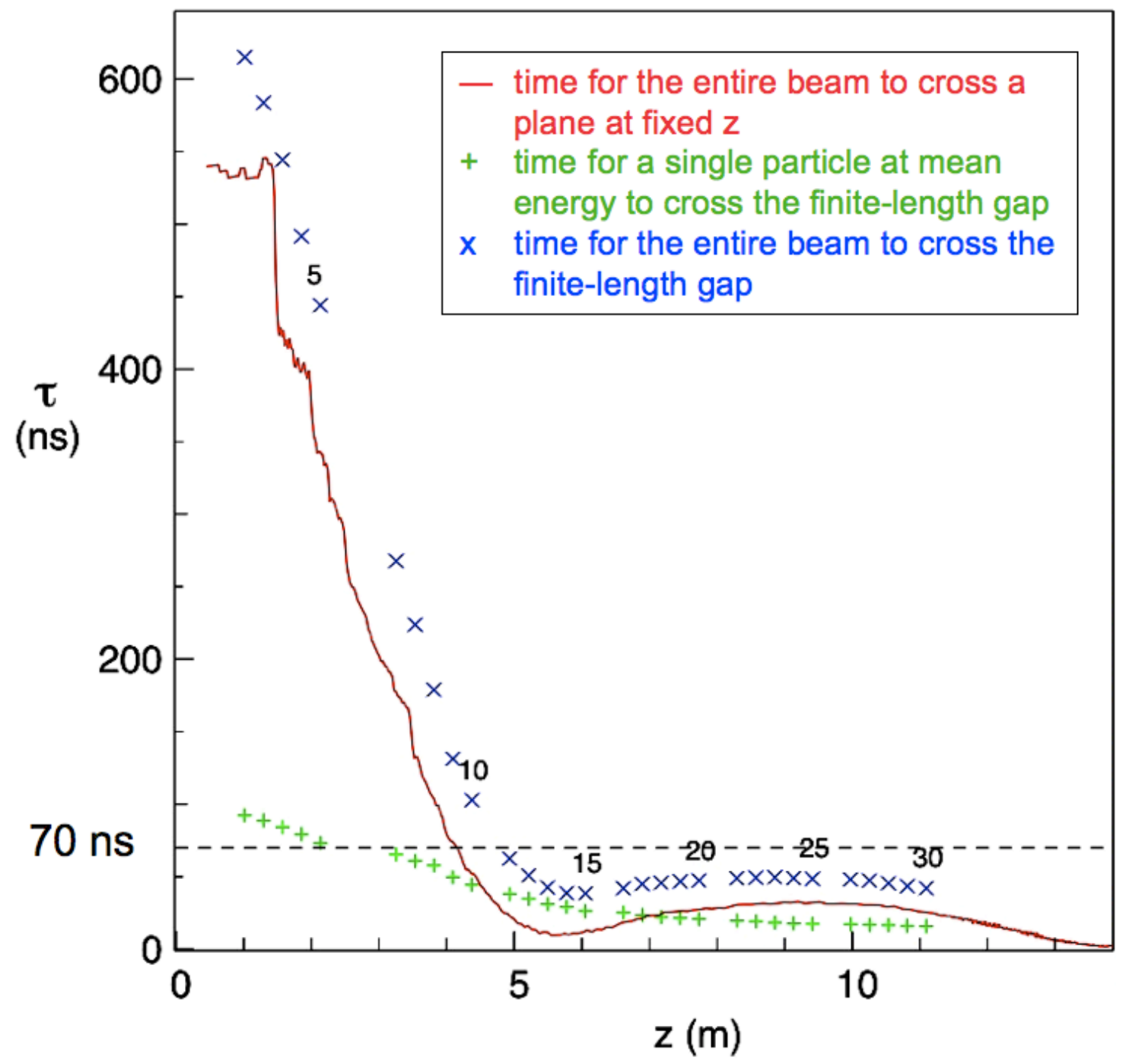

FIG. 2: Pulse duration vs. $z$; the key measure is the time for the entire beam to traverse a gap's extended fringe field from entrance to exit.

generated by solving appropriate circuit equations, producing the nearly "triangular" (linearly rising from zero) pulses seen in Fig 3. Similar circuits are being studied to form the tilt waveforms in the first two blocks. The low-voltage highly shaped "ear" waveforms may require programmable circuits like those developed by First Point Scientific. Figure 4 shows the evolution of the beam phase space and line-charge-density profile; its final panel shows the beam when its centroid is at the "best longitudinal focus" plane. This plane is estimated by an RMS measure [13] and then refined by 


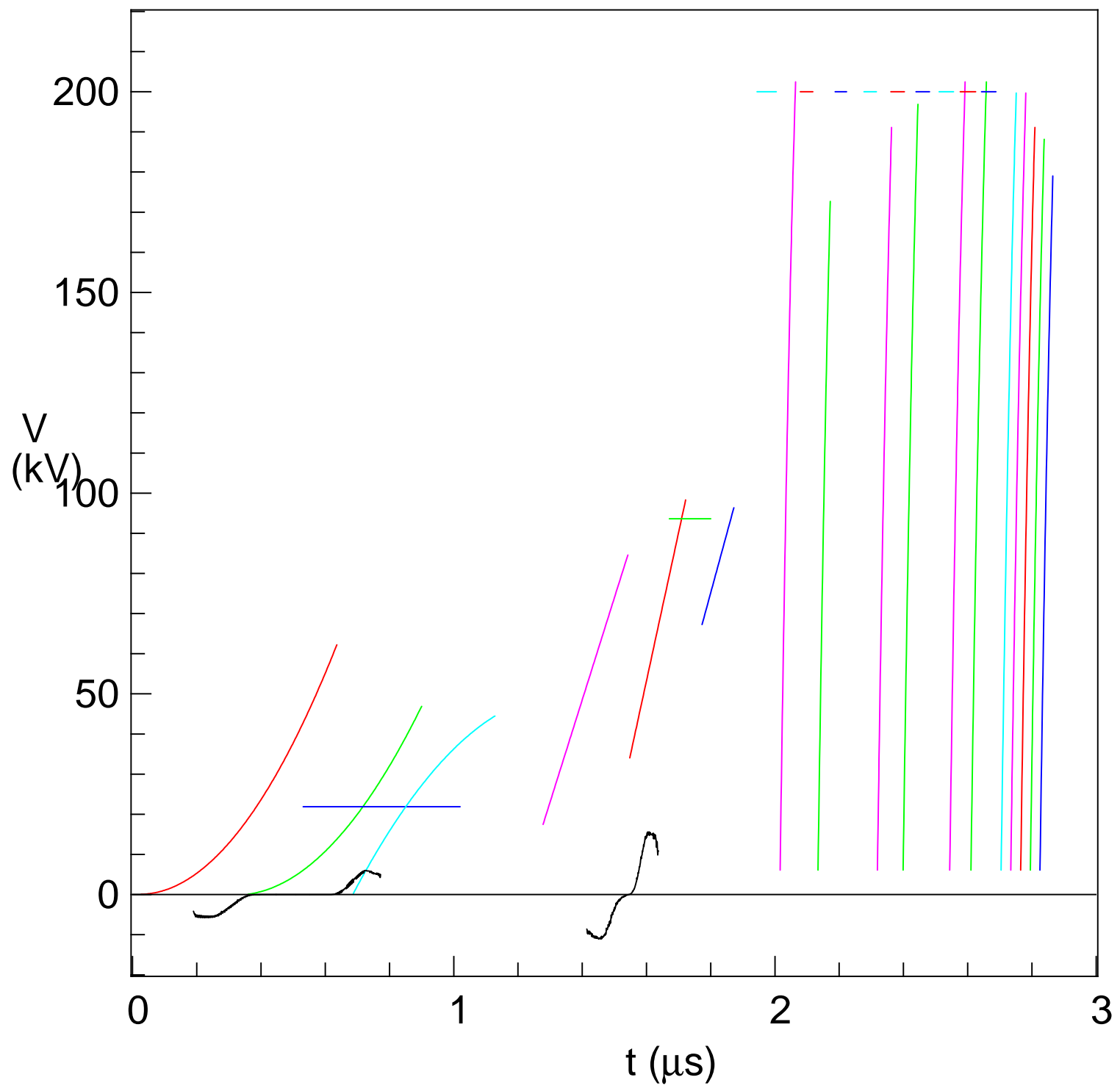

FIG. 3: Waveforms for accelerating gaps as developed using 1-D simulations.

explicitly finding that plane through which the most current flows in an optimal 1-ns window.

While these 1-D results are encouraging, the model necessarily omits radial variation of the acceleration and space charge fields, as well as the physics of transverse confinement and focusing. Initial axisymmetric $(\mathrm{r}, \mathrm{z})$ simulations using Warp validate the 1-D model. When we use the acceleration fields shown in Fig. 3 and an initial Warp 
beam with the same line-charge, energy, and longitudinal profile, no particles are lost and the final energy and pulse duration are similar to the 1-D results of Fig. 4. As shown in Fig. 5, the transverse dynamics is well behaved although there is some envelope mismatch; the normalized transverse emittance grows from 0.9 to $1.2 \mathrm{~mm}-\mathrm{mr}$ in the accelerator. Figure 6 shows snapshots taken at the same time from 1-D and Warp runs, for similar conditions; the gross beam characteristics, and the energy, are quite similar, but the details of the phase space and line-charge density profile differ. Some fine-tuning of the parameters of the 1-D model (e.g., the scale-length for transverse field fall-off used in the Poisson equation that is solved to obtain the space-charge field [14]) might improve the agreement, but in any event final adjustments of waveforms wil be done in Warp. This early Warp run yields somewhat poorer compression than was obtained in 1-D, with a peak current about $30 \%$ lower. Adjustments of the strengths of the 2-3 $\mathrm{T}$ confining solenoids in the accelerator and the final 8-15 $\mathrm{T}$ focusing solenoid are ongoing.
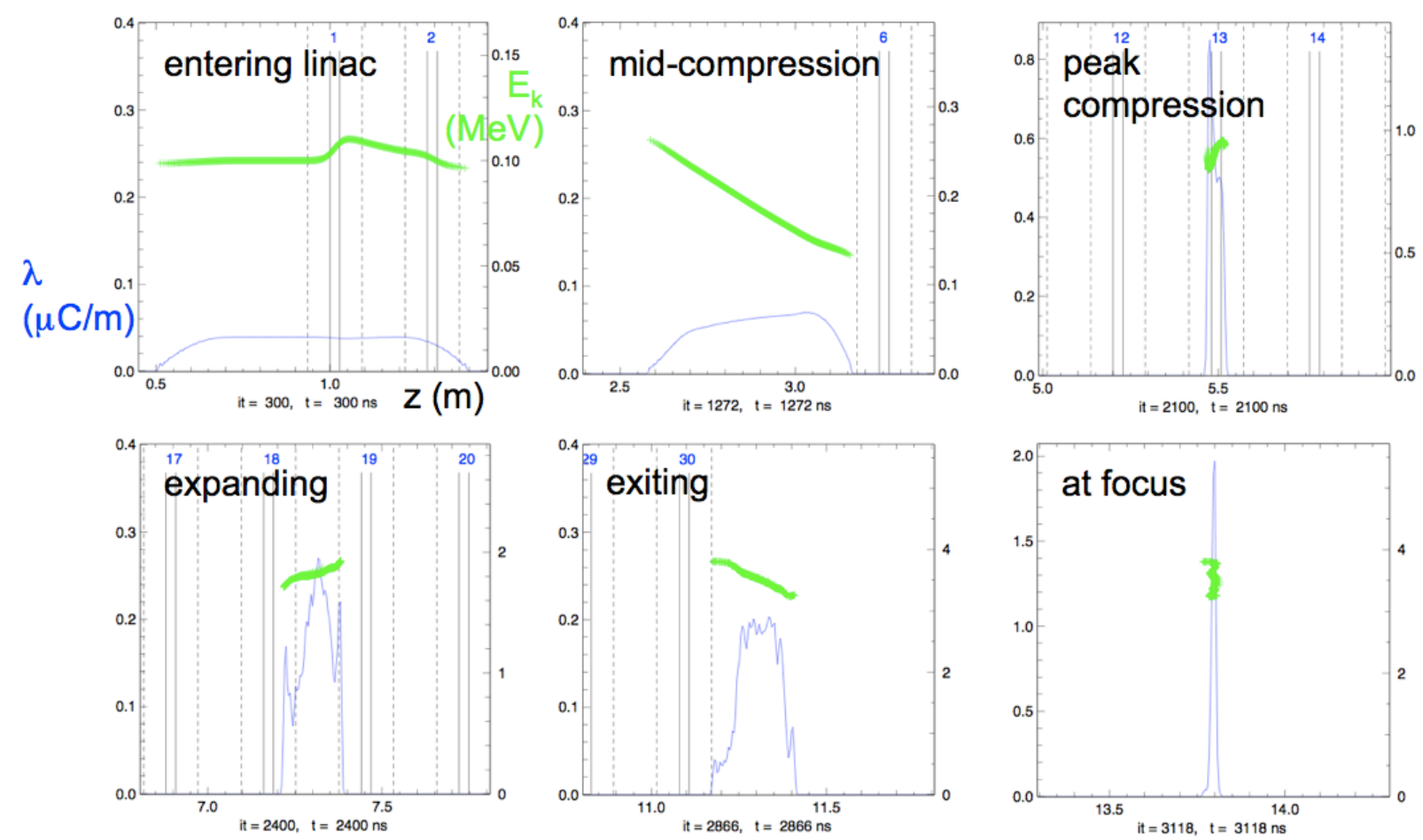

FIG. 4: Snapshots from 1-D code at selected times: longitudinal phase space of energy vs. $z$ position (right ordinate scale, individual markers); and $\lambda$ (averaged over $\sim 4 \mathrm{~mm}$ ) (left ordinate scale, thin trace).

Efforts are in progress to use Warp to simulate the NDCX-II beam from the source to 
the target plane. These simulations are more challenging than the run described above (which was initiated with a uniform-energy beam) because the ion beam already has a significant energy variation by the time it reaches the first gap, due to both transit-time effects in the diode and the longitudinal space-charge field. To remove most of this initial energy nonuniformity, we use an ear cell immediately after injection. Initializing the 1-D code with a beam containing an idealized energy variation, we have developed an acceleration schedule that produces a beam with nearly the same final parameters as those found for the initially monoenergetic beam, but with a small fraction of straggling ions at lower energies. Early Warp results with this new schedule give a beam with the correct final energy and a somewhat smaller head-to-tail velocity tilt; we are presently working to improve this case.
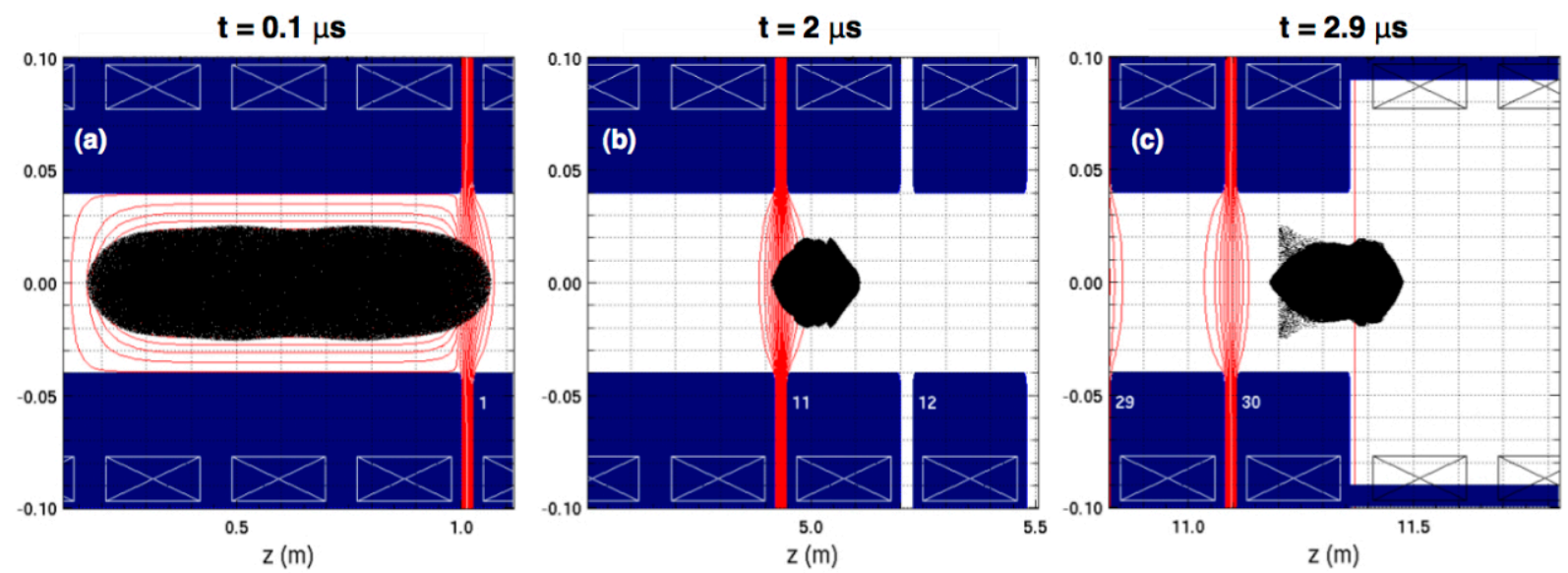

FIG. 5: Views of beam in $(x, z)$ projection at selected times, as simulated using Warp. The inductive accelerating field is treated, to good aproximation, as electrostatic, so contour lines denote equipotentials.

As the beam leaves the accelerator it is neutralized and allowed to drift-compress until it passes through a final strong solenoid and is focused onto the target. Extensive calculations of both the beam dynamics of this process and the injection of plasma have been carried out using the LSP code. For an overview and a description of an integrated calculation for NDCX-I, see [15]. Reference [15] also details the "Lee" accelerating-gap field model employed in our 1-D studies; we use a single-term approximation because the fringing length is much greater than the physical gap length. For an overview of LSP-based plasma injection studies, see [16]. Warp is also being used for neutralizedbeam and plasma injection studies relevant to both NDCX-I and NDCX-II. As an example, Figure 7 shows a snapshot from a simulation of plasma injection by the FCAPS sources in NDCX-I; colors denote the plasma density.

Our 1-D simulations suggest that the beam is insensitive to waveform details after 

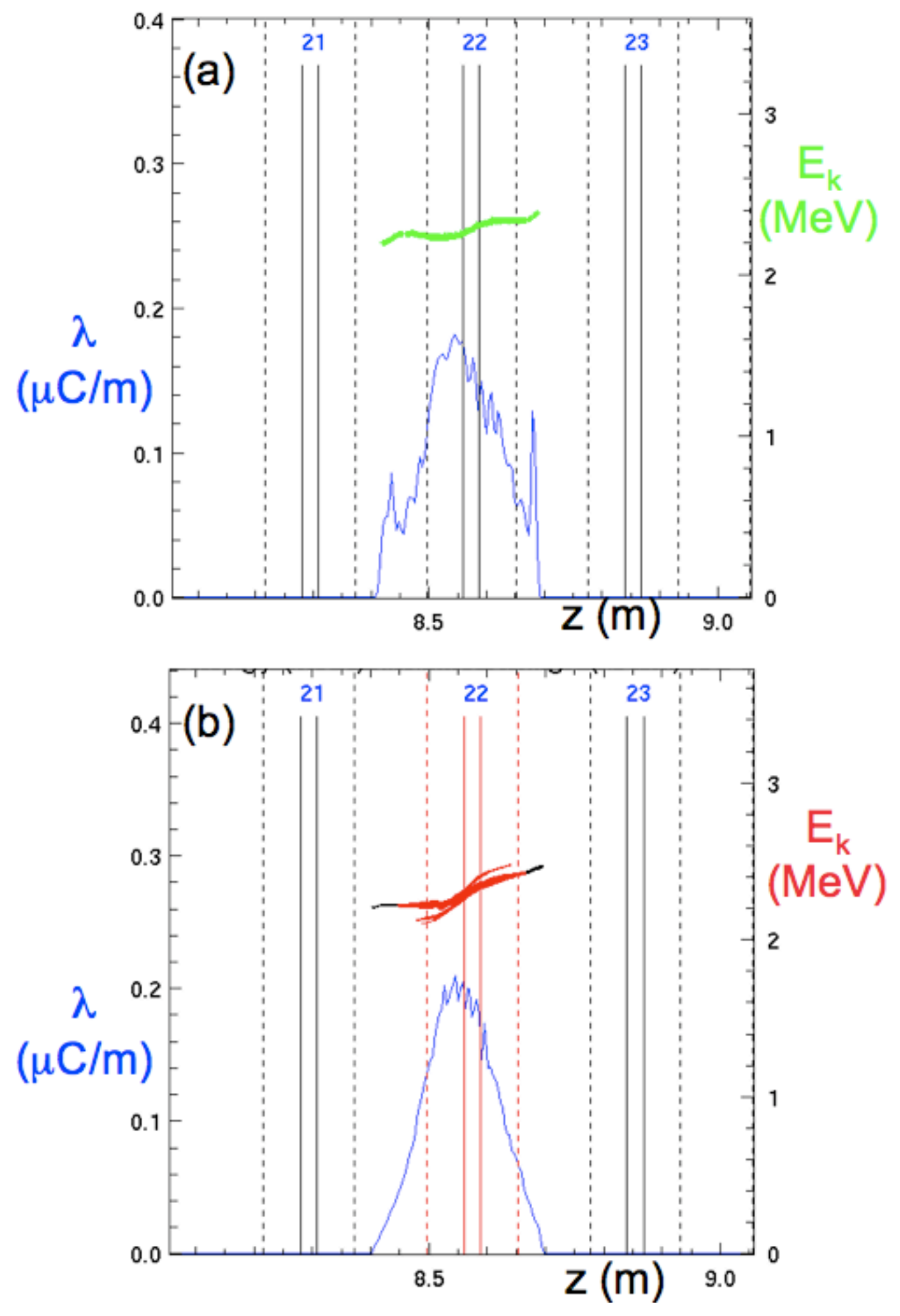

FIG. 6: $\left(E_{k}, z\right)$ phase space and line-charge density at $2.58 \mu$ s from (a) 1 -d code run, and (b) Warp run. 

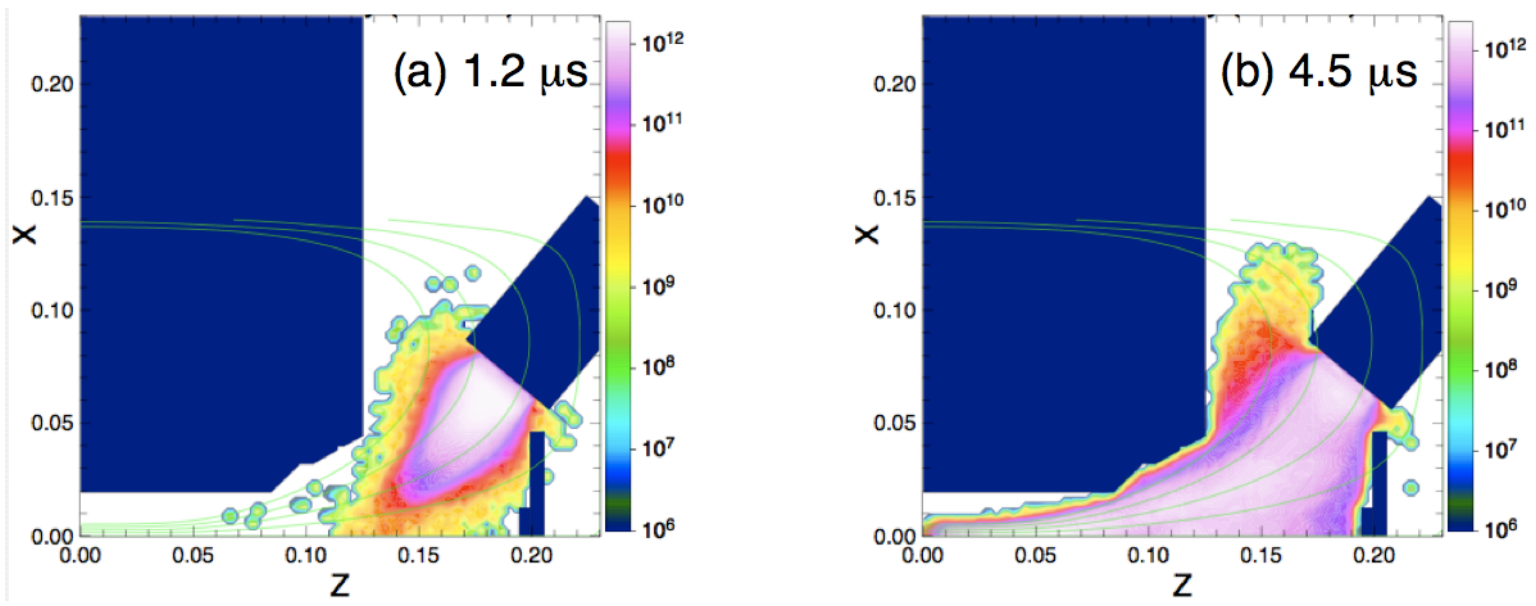

FIG. 7: Frames from a Warp simulation of plasma injection at (a) $1.2 \mu \mathrm{s}$ and (b) $4.5 \mu \mathrm{s}$.

the initial compression. However, tolerances for waveform timing and voltage need be developed with 2-D Warp studies. 3-D studies will develop steering techniques and establish tolerances for the alignment and field strength of solenoids. We will use simulations and analysis to develop an optimized final focusing system, accounting for the dependence of the size and profile of the focal spot upon the velocity tilt, focusing angle, chromatic aberration, and properties of the neutralizing background plasma [17]. The ability of time-dependent focusing to correct for chromatic effects [18] in NDCX-II will be quantified, and a practical design worked out. We will explore improved means of plasma injection and control for neutralized compression and focusing; systems based on technologies other than the existing FCAPS may prove superior.

While to date we have concentrated on an initial NDCX-II configuration aimed at WDM studies, a smaller amount of work has considered the modifications necessary to produce higher kinetic energies, multiple pulses, and/or longer pulses with ramped energy, to study energy coupling and hydrodynamic stability in ion direct drive. NDCX-II promises to be a flexible facility capable of supporting a wide range of experiments.

\section{Acknowledgments}

This work was performed under the auspices of the U.S. Department of Energy by Lawrence Livermore National Laboratory under Contract DE-AC52-07NA27344, by Lawrence Berkeley National Laboratory under ContractDE-AC02-05CH11231 and by the Princeton Plasma Physics Laboratory under Contract DE-AC02-76CH03073. 
[1] B. G. Logan, et al., Nucl. Instr. and Meth. A 577 (2007) 1.

[2] Proc. 17th Int. Sympos. on Heavy Ion Inertial Fusion (HIF2008), Tokyo, Aug. 4-9, 2008, hereinafter referred to as these Proceedings.

[3] B. G. Logan, et al., these Proceedings.

[4] J. E. Coleman, et al., Proc. 2007 Part. Accel. Conf.; http://accelconf . web.cern.ch/AccelConf/.

[5] P. A. Seidl,, these Proceedings.

[6] W. M. Sharp, et al., these Proceedings.

[7] E. Henestroza, et al., Proc. 2005 Part. Accel. Conf.; http://accelconf . web.cern.ch/AccelConf/.

[8] Workshop on Accelerator Driven Warm Dense Matter Physics, Pleasanton, CA, Feb. 2006: http://hifnews.1bl.gov/HIFSNewsNo3_06.pdf and http://hifweb.lbl.gov/public/AcceleratorWDM/proceedings/.

[9] R. J. Briggs, Phys. Rev. ST Accel. Beams 9, 060401 (2006).

[10] A. Friedman, et al., Proc. 2007 Part. Accel. Conf.; http://accelconf.web.cern.ch/AccelConf/.

[11] W. L. Waldron, et al., Proc. 2007 Part. Accel. Conf.; http://accelconf.web.cern.ch/AccelConf/.

[12] C. Y. Ling, et al., these Proceedings.

[13] A. Friedman, "Some kinematic aspects of neutralized drift compression," LLNL Research Note LLNL-TR-402447, March 18, 2008.

[14] J. J. Barnard, et al. Proc. 1993 Part. Accel. Conf.; http://accelconf.web.cern.ch/AccelConf/.

[15] D. R. Welch, et al., Phys. Rev. ST Accel. Beams 11, 064701 (2008).

[16] A. B. Sefkow, R. C. Davidson, and E. P. Gilson, Phys. Rev. ST Accel. Beams 11, 070101 (2008).

[17] J. J. Barnard, et al., Proc. 2005 Part. Accel. Conf.; http://accelconf . web.cern.ch/AccelConf/.

[18] E. P. Lee, Proc. IFE Science and Technology Strategic Planning Workshop, San Ramon, CA, April 24-27, 2007; http://ifeworkshop.llnl.gov/proceedings.html Part 5, Pp. 109-124. 